\title{
Comparison of transfection efficiency of polymer-based and lipid-based transfection reagents
}

\author{
Rahimi $\mathrm{P}^{1}$, Iranpur Mobarakeh $\mathrm{V}^{1}$, Kamalzare $\mathrm{S}^{1}$, SajadianFard $\mathrm{F}^{1}$, Vahabpour $\mathrm{R}^{2}$, Zabihollahi $\mathrm{R}^{1}$
}

Department of Hepatitis and AIDS, Pasteur Institute of Iran, Tehran, Iran. pooneh5376@yahoo.com

\section{ABSTRACT}

OBJECTIVES: In this study, the optimal dose of Lipofectamine 3000 and Turbofect to transfect adherent cell lines such as CHO-K1 and HEK293 cells in comparison with non-adherent H9T-cells with pEGFP-N1 and pCDH was identified.

BACKGROUND: Lipofectamine 3000 is a new transfection reagent which is claimed to be more efficient than other transfection reagents like Turbofect. Transfection efficiency could be affected by the nature of target cell line and vector.

METHODS: Transfection efficiency and cytotoxicity of each reagent was identified by using flow cytometry and XTT assay, respectively.

RESULTS: Lipofectamine 3000 was more efficient in transfecting pCDH, while Turbofect was more efficient in separate transfection of CHO-K1 and HEK293 with pEGFP-N1. Lipofectamine 3000 could be cytotoxic in transfecting H9T-cells with pCDH. Also, H9T-cells were not sufficiently transfected with each plasmid vector by using each Lipofectamine 3000 and Turbofect. Turbofect had less cytotoxicity effect on all three cell lines than Lipofectamine 3000 .Transfection of suspended cells like H9T-cells by using Lipofectamine 3000 and Turbofect would not result in sufficient transfection.

CONCLUSION: Lipofectamine 3000 is the best choice for transfection of CHO-K1 and HEK293 with pCDH while Turbofect is preferably used in transfecting these cell lines with pEGFP-N1 (Tab. 1, Fig. 2, Ref. 26). Text in PDF www.elis.sk.

KEY WORDS: cell transfection, pCDH, pEGFP-N1, Lipofectamine 3000, Turbofect.

\section{Introduction}

Modification or manipulation of organisms in order to understand better the functions of genes and finding more powerful treatment tools for genetic disorders (gene editing/gene therapy) and life improvement are considered as one of the most human beings' desirable ambitions. To achieve this, "genetic engineering" with various techniques such as gene cloning, transformation, transfection, and transduction have been developed while the recent innovation referred to as gene editing (CRISPER) is causing a revolution in science. Gene cloning methods result in "recombinant DNA" that contains two or more DNA molecules or genes of which some are combined. This recombinant DNA needs a proper host to be transferred to and get support for its propagation

${ }^{1}$ Department of Hepatitis and AIDS, Pasteur Institute of Iran, Tehran, Iran, and ${ }^{2}$ Medical Lab Technology Department, School of Allied Medical Sciences, Shahid Beheshti University of Medical Sciences, Tehran, Iran

Address for correspondence: P. Rahimi, Department of Hepatitis and AIDS, Pasteur Institute of Iran, 12 Farvardin Ave, Enghelab Square, Tehran, Iran.

Phone: +982166969291

Acknowledgement: The financial support was provided by Iran National Science Foundation under the grant No. 93013827. for further studies. Introducing an exogenous recombinant nucleic acid to a host cell such as that of bacteria, insects or mammals is possible by using physical, chemical and biological methods such as transfection reagents, calcium phosphate, polybrene, and polyethyleneimine which, results in producing new cells with genetic modifications (1-6). Dendrimers, DEAE-dextran or cationic lipidbased reagents are supposed to coat the negatively charged DNA/ RNA, creating an overall positive charge or neutralizing the molecule and introducing it to the negatively charged cell membrane, thus being ideal for transfection of different types of cells such as primary cells and difficult-to-transfect cells (3).

There are many kinds of transfection reagents such as lipidbased and polymer-based transfection reagents. Cationic lipid vesicles or "liposomes" are known to be efficient transfection reagents able to interact with exotic DNA and facilitate its delivery into the cell through fusion with cell membrane. This is possible even in cell types that are resistant to calcium phosphate or DEAE-dextran $(7,8)$. This transfection reagent can be used for both long-term and stable expression experiments that need integration of introduced DNA into the chromosome or transient expression studies such as episomal maintenance. Also, it can be used for in vivo experiments of transferring DNA or RNA to animals and humans $(7,8)$. Choosing a more suitable transfection reagent depends on some criteria such as type of host cell line, type of 
target molecule considered to be transferred to a cell line or aim of transfection which means it is either a transient transfection or a stable one. However, optimizing the transfection conditions could be useful in achieving highest efficiency. Lipid-mediated transfection has the capability of adaptation to high throughput systems and is one of the most applicable transfection methods in different studies mainly because of its ease of use and low cost (9). In this study, we compare the impact of Lipofectamine $\AA 3000$ and Turbofect in efficient transfection of CHO-K1, HEK293, and H9T-cells with pEGFP-N1 and pCDH/CMV-GFP-Puro plasmids by measuring the expression level of enhanced green fluorescent protein (EGFP) in transfected cells.

\section{Materials and methods}

\section{Cell types and cell culture}

In this study H9T-cells were cultured as suspended cells and CHO-K1 and HEK293 were used as adherent cells while all cells were purchased from National Cell Bank of Iran (NCBI), Pasteur Institute of Iran. All cell lines were cultured in $25 \mathrm{~cm}^{2}$ filter cap cell culture flasks separately containing the appropriate cell culture media according to each cell line. H9T-cells were grown in RPMI medium $\left(\mathrm{Gibco}^{\mathrm{TM}}\right.$, Fisher Scientific, USA) with $\mathrm{pH}=7.2$ containing $10 \%$ fetal bovine serum (FBS, biosera, France) and $1 \%$ of penicillin-streptomycin (biosera, France). CHO- K1 cells were grown in $5 \mathrm{ml}$ of Ham's F-12 medium (Caisson, USA) with $\mathrm{pH}=7.2$, and fetal bovine serum (FBS, biosera, France) with final concentration of $10 \%, 1 \%$ of penicillin-streptomycin (biosera, France). To culture HEK293 cell line, DMEM High Glucose medium with $\mathrm{pH}=7.2$ which contained $1 \%$ of penicillin-streptomycin (biosera, France), and $10 \%$ fetal bovine serum (FBS, biosera, France) was used. The cell culture flasks were maintained in an incubator at $37^{\circ} \mathrm{C}$ with $5 \% \mathrm{CO}_{2}$ until the cells reached density of 60-80\%. To suspend CHO-K1 and HEK293 cell lines, $0.5 \mathrm{ml}$ of 10X Trypsin-EDTA (Caisson, Trypsin $0.5 \%, 10 \mathrm{X}$ ) was used and both cell lines were successfully suspended through pipetting and then transferred to the 24-well cell culture plates. H9T-cells were also transferred to cell culture plates. All cell culture plates were incubated at $37{ }^{\circ} \mathrm{C}$ with $5 \% \mathrm{CO}_{2}$.

\section{Plasmids amplification}

Viral vector pCDH-CMV-GFP-Puro with $8.2 \mathrm{~Kb}$ was purchased from System Biosciences, USA. This lentivector with 8.2 $\mathrm{Kb}$ contains CMV promoter and GFP biomarker which could be detected in transfected cells by using flow cytometry and fluorescent microscopy. The pEGFP-N1 plasmid with $4.7 \mathrm{~Kb}$ as a nonviral vector was purchased from Clontech, USA. Each plasmid vector was transferred separately to a competent E. coli DH5a strain by using heat shock method. Transfected E. coli bacteria were grown overnight on LB agar medium containing kanamycin sulfate at concentration of $50 \mathrm{mg} / \mathrm{ml}$ (Merck, Germany) at 37 ${ }^{\circ} \mathrm{C}$. Then, both plasmid vectors were extracted by using Plasmid DNA Purification Maxi kit (Qiagen, USA) according to the manufacturers 'instructions, and the integrity of isolated plasmids was investigated by using $1 \%$ agarose gel electrophoresis analysis.
These two vectors contain GFP, a strong biomarker which can be detected easily in transfectants.

\section{Cell transfection procedures}

In this study, two most famous reagents, namely Lipofectamine 3000, a cationic lipid-based transfection reagent, and Turbofect, a cationic polymer-based transfection reagent, were used (Thermo Fisher Scientific, USA). At first, the optimum ratio of each plasmid vector (pEGFP-N1 and $\mathrm{pCDH}$ ), and each transfection reagent must be identified. Therefore, $1 \mu \mathrm{g}$ of each plasmid vector was mixed with volumes of 1,2 , and $3 \mu$ of each transfection reagent according to their related manufacturers ' instructions. Each mixture was used to transfect each of three cell lines separately and the best ratio was identified in a mixture of $1 \mu \mathrm{g}$ of each plasmid vector with $2 \mu 1$ of each transfection reagent. This ratio was used for transfecting each cell line which was cultured in two 24-well cell culture plates to be applied for evaluating the efficiency of each transfection reagent separately. The experiment was done in triplicate for each plasmid vector. There were also non-transfected cells for each cell line as a negative control. The confluency of $2 \times 10^{5}$ cells was reached in each well for H9T-cell culture and $10^{5}$ cells for CHO-K1, and HEK293 cell lines. Turbofect transfection mixture was prepared by adding $1 \mu \mathrm{g}$ of each plasmid vector to $100 \mu 1$ of serum free medium; Opti-MEM (Gibco, Thermo Fisher Scientific, USA) and adding $2 \mu 1$ of Turbofect reagent. A volume of $100 \mu 1$ of each mixture was added to each well of cell culture plates while the plates were being shook gently. Lipofectamine 3000 was initiated by adding separately $1 \mu \mathrm{g}$ of each plasmid vector and $2 \mu \mathrm{l}$ of Lipofectamine 3000 to $25 \mu \mathrm{l}$ of serum-free medium, Opti-MEM (Gibco, Thermo Fisher Scientific, USA). Finally, $2 \mu 1$ of P3000 reagent (provided in Lipofectamine 3000 kit) was added to each mixture and mixed gently by pipetting. The plates were incubated at $37^{\circ} \mathrm{C}$ and $5 \% \mathrm{CO}_{2}$ for $48 \mathrm{~h}$. GFP emission of each

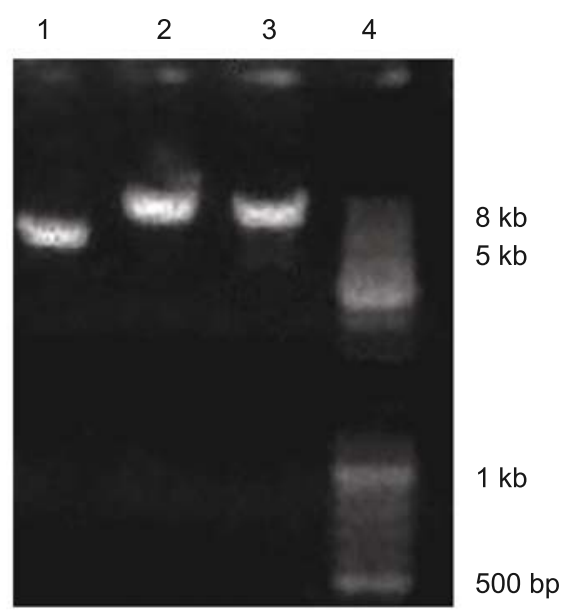

Fig. 1. Gel electrophoresis of pEGFP-N1 and pCDH plasmids. Confirmation of integrity of extracted plasmid vectors; $\mathrm{pEGFP-N1}$ and pCDH on $1 \%$ agarose gel electrophoresis. Lane 1: pEGFP-N1 plasmid with 4.7 bp. Lanes 2 and 3: pCDH plasmid with 8.2 bp. Lane 4: 500bp DNA ladder. 
plasmid vector was analysed in each well of all cell culture plates by flow cytometry.

\section{XTT cell proliferation assay}

Viability of each transfected cell line was evaluated by using cell proliferation kit II (XTT) (Roche Diagnostics, Germany), and the absorbance of each well was measured at 8,24 and 48 hours after transfection at $450 \mathrm{~nm}$ and $630 \mathrm{~nm}$ according to the manufacturer`s instruction.

\section{Statistical analysis}

Data obtained from each triplicated test for each transfection reagent were statistically analysed using SPSS Software Version 22, and Pair-Samples T-Test. The results were considered statistically significant when the $\mathrm{p}$ value was equal to or lower than 0.05 .

\section{Results}

The integrity of both plasmids, i.e. pCDH and pEGF-N1, was confirmed on gel electrophoresis as shown in Figure 1.

The mean results of all triplicated tests for each transfection reagent obtained from flowcytometry analysis are summarized in Table 1.

As shown in Table 1, the best transfection efficiency was achieved with Turbofect to transfect CHO-K1 cells with pEGFP$\mathrm{N} 1(74 \%)$ while the most insufficient transfection results were obtained from transfecting H9 T-cells with $\mathrm{pCDH}$ plasmid vector by using Turbofect $(0.7 \%$ ). Both Lipofetamine 3000 and Turbofect showed the insufficient transfection efficiency to transfect H9Tcells by using $\mathrm{pCDH}$ with rates $1.6 \%$ and $0.7 \%$, respectively, and pEGFP-N1 with rates $1 \%$ and $2.1 \%$, respectively. Also, in comparison with Lipofectamine 3000 Turbofect was more efficient to transfect adherent cell lines (CHO-K1 and HEK293) with pEGFPN1 (74 \%, 59\% vs $55 \%$ and $52 \%$, respectively) and contrarywise, in comparison with Turbofect, Lipofectamine 3000 showed higher transfection efficiency to transfect adherent cell lines with pCDH ( $64 \%$ and $53 \%$ vs $56 \%$ and $44 \%$, respectively). Figure 2 depicts the results of flowcytometry analysis of one of the triplicate tests of each transfected cell line with each plasmid vector $(\mathrm{pCDH}$ and

Tab. 1. The Mean results of flow cytometry analysis of each transfected cell line by using Turbofect and Lipofectamine 3000 separately with each pEGFP-N1 and $\mathrm{pCDH}$ plasmid vector.

\begin{tabular}{lccc}
\hline Cell line & Plasmid vector & $\begin{array}{c}\text { Lipofectamine 3000 } \\
\text { Mean } \%\end{array}$ & $\begin{array}{c}\text { Turbofect } \\
\text { Mean } \%\end{array}$ \\
\hline \multirow{2}{*}{ H9 T-cell } & pEGFP-N1 & 1 & 2.1 \\
& pCDH & 1.6 & 0.7 \\
\hline \multirow{2}{*}{ CHO-K1 } & pEGFP-N1 & 55 & 74 \\
& pCDH & 64 & 56 \\
\hline \multirow{2}{*}{ HEK293 } & pEGFP-N1 & 52 & 59 \\
& pCDH & 53 & 44 \\
\hline
\end{tabular}

The best transfection efficiency was achieved in using Turbofect to transfect CHOK1 cells with pEGFP-N1 (74\%) while the most insufficient transfection results were obtained from transfecting $\mathrm{H} 9 \mathrm{~T}$-cells with $\mathrm{pCDH}$ plasmid vector by using Turbofect $(0.7 \%)$. Both Lipofetamine 3000 and Turbofect showed insufficient transfection efficiency in transfecting H9T-cells by using pCDH with $1.6 \%$ and $0.7 \%$, respectively and pEGFP-N1 with $1 \%$ and $2.1 \%$, respectively.

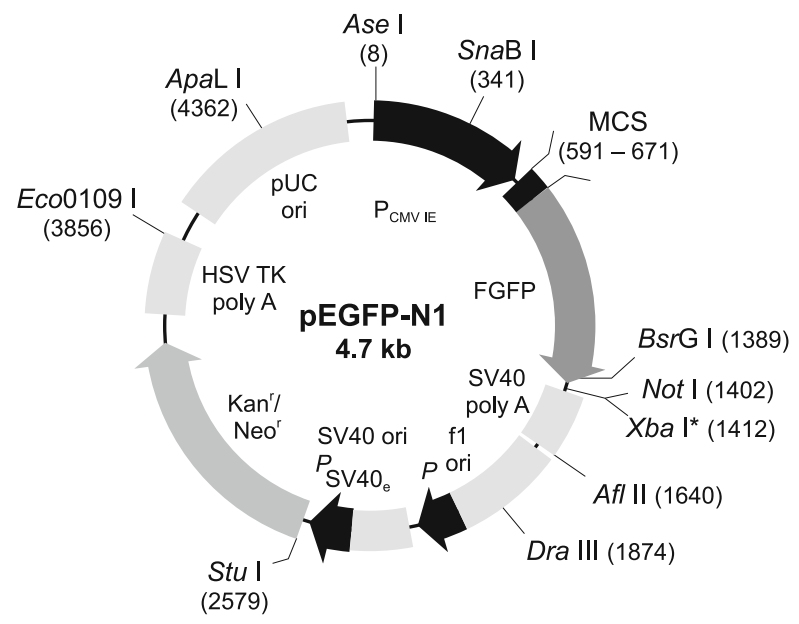

Fig. 2. Measurements of green fluorescent protein (GFP) emission as a biomarker in pEGFP-N1 and pCDH vectors. Green fluorescent protein (GFP) was expressed from each transfected cell line by using flow cytometry analysis. Transfection of H9T-cells with each pEGFPN1 and pCDH by using Lipofectamine 3000 and Turbofect separately showed poor results. Lipofectamine 3000 and Turbofect were effective in transfecting both CHO-K1 and HEK293 as adherent cell lines with each pEGFP-N1 and pCDH plasmid vector separately.

pEGFP-N1) by separately using each Lipofectamine 3000 and Turbofect transfection reagent.

In XTT assay, Lipofectamine 3000 showed statistically significant cytotoxicity effect on transfecting the H9T-cells with pCDH in comparison with CHO-K1 and HEK293 cells transfected with $\mathrm{pCDH}(0.23,0.867$ and 0.824 , respectively; $\mathrm{p}<0.05)$. There was no significant difference in cytotoxicity between cell lines transfected with pEGFP-N1 by using Lipfectamine $3000(p>0.05)$. The results of using Turbofect to transfect each cell line with $\mathrm{pCDH}$ or pEGFP-N1 showed no statistically significant cytotoxicity effect on either of three cell lines $(\mathrm{p}>0.05)$.

\section{Discussion}

Cation-lipid-based transfection reagents have been used widely in many transfection experiments because of their characteristic property of forming liposomes in aqueous media with an ability to fuse with the host cell membrane resulting in entrance of target DNA into the cytoplasm or nucleus (6-11). Many studies have revealed that the efficiency of transient transfection could be increased by using serum-free medium such as Opti-MEM to increase foreign DNA uptake. This effect has been shown particularly in application of cationic nanoparticles that are taken up by cells via endocytosis (12). In this study, although both transfection mixtures were prepared using Opti- MEM, the transfection efficiency was significantly low in H9 T-cells. These observations could result from weak endocytosis in H9T-cell or weak promoter inductions of viral vectors such as $\mathrm{pCDH}$ in suspended cells like 


\section{1-705}

H9 T-cells (13-15). There are some important considerations for optimal transfection using Lipofectamine 3000 and Turbofect because transfection is a manipulating method which may bring about some cell deaths and there are several factors that affect the outcomes. Cellular density at the time of transfection is an important criterion that leads to variations in expression of transfected gene/s and the best cellular confluence is known to be 90-95\%. Inhibition of cell growth through impaired metabolism occurs when the density is too high. In contrast, low density could make the cell culture vulnerable after transfection as described by Kim and Eberwine (1). In this study, cellular density for H9 T-cells was 200,000 and it was 100,000 for each CHO-K1 and HEK293 cells, thus it was in a suitable range reported in different transfection studies (16).

In most studies, transfection efficiency is evaluated using biomarkers such as luciferase and $\operatorname{GFP}(7,8,10,11,17,18)$. In this study, GFP in pEGFP-N1 and pCDH plasmid vectors were used as strong biomarkers for evaluating flowcytometry. Limitation of any possible undesirable effects on cells such as cellular toxicity is of particular concern in each transfection experiment while the achievement of high transfection efficiency through optimization of the reagent/DNA ratio is the most important goal. However, cell membrane composition, and other conditions such as $\mathrm{PH}$ have a strong influence on transfection outcomes according to Kim and Eberwine (1). Herein, the optimal ratio of each transfection reagent/ each plasmid vector was identified as $1 \mu \mathrm{g}$ of each plasmid vector and $2 \mu \mathrm{l}$ of each transfection reagent. The most and the least transfection efficiency ( $74 \%$ and $0.7 \%$, respectively) were obtained by using Turbofect to transfect CHO-K1 cells with pEGFP-N1 and $\mathrm{H} 9 \mathrm{~T}$-cells with pCDH plasmid vector, respectively. Herein, separate transfection of H9T-cells with pCDH and pEGFP-N1 by using each transfection reagent resulted in poor transfection and when compared with the results of transfecting adherent cell lines with $\mathrm{pCDH}$ and $\mathrm{pEGFP}-\mathrm{N} 1$ by using each reagent separately, the difference was statistically significant $(p>0.5)$. Interestingly, in comparison with Lipofectamine 3000, Turbofect was more efficient to transfect CHO-K1 and HEK293 with rates $74 \%$, and 59 $\%$, respectively with pEGFP-N1. It has been known that Liposomal transfection reagents such as Lipofectamine are not as safe as polyethylenimine or polyamine-based reagents for cells (9).

Lipofectamine 2000 has been used in many studies and is known as an effective transfection system. Therefore, there are many reports of its application in different studies with up to 80 $\%$ efficiency accompanied with significant toxicity in different cell lines such as primary cells, fibroblasts, and stem cells (9). According to the manufacturers' instruction, Lipofectamine 3000 is a new generation with some improvements which are expected to be more efficient with less cytotoxicity in comparison with Lipofectamine 2000. The results of this study revealed that Lipofectamine 3000 showed a significant cytotoxic effect on transfected H9T cells with pCDH (0.23) in comparison with CHO-K1 and HEK293 cells $(0.867)$. In contrast to Turbofect, there was no statistically significant cytotoxicity effect on either of three cell lines. These findings are consistent with other studies on cytotoxicity effect of Turbofect (8). Although there are many reports about the cellular toxicity of Lipofectamine 2000 in comparison with other transection reagents such as Turbofect, there are just few published studies on toxicity of Lipofectamine $3000(9,19,20-23)$. In one study, Lipofectamine CRISPRMAX was compared with Lipofectamine 3000 and Lipofectamine RNAiMAX, and significant efficiency with less toxicity of Lipofectamine CRISPRMAX was shown (12, 23-26). In this study, Lipofectamine 3000 showed significant cellular toxicity on H9T-cells after transfection with pCDH vector. Thus, according to our results, Turbofect was safer for transfecting both suspended and adherent cells with viral and non-viral vectors. However, transfecting H9T-cells by using each reagent resulted in a significantly poor transfection in comparison with two adherent cell lines (CHO-K1 and HEK293). The comparison of transfection efficiency showed that Lipofectamine 3000 was more efficient in transfecting $\mathrm{pCDH}$ plasmid vector while Turbofect showed higher efficiency in transfecting CHO-K1 and HEK293 with pEGFP-N1.

\section{References}

1. Kim TK, Eberwine JH. Mammalian cell transfection: the present and the future. Anal Bioanal Chem 2010; 397 (8): 3173-3178. DOI: 10.1007/ s00216-010-3821-6.

2. Kawai S, Nishizawa M. New Procedure for DNA Transfection with Polycation and Dimethyl Sulfoxide. Mol Cell Biol 1984; 4 (6): 1172-1174.

3. Wyman TB, Nicol F, Zelphati O et al. Design, Synthesis, and Characterization of a Cationic Peptide That Binds to Nucleic Acids and Permeabilizes Bilayers. Biochemistry 1997; 36 (10): 3008-3017. DOI: 10.1021/ bi9618474.

4. Boussif O, Lezoualc'h F, Zanta MA et al. 1995. A versatile vector for gene and oligonucleotide transfer into cells in culture and in vivo: polyethylenimine. Proc Natl Acad Sci USA 1995; 92 (16): 7297-7301. PMID: 7638184.

5. Haensler J, Francis C, Szoka Jr. Polyamidoamine cascade polymers mediate efficient transfection of cells in culture. Bioconjug Chem 1993; 4 (5): 372-379. DOI: 10.1021/bc00023a012.

6. Kukowska-Latallo JF, Bielinska AU, Johnson J, Spindler R, Tomalia DA, Baker Jr JR. Efficient transfer of genetic material into mammalian cells using Starburst polyamidoamine dendrimers. Proc Natl Acad Sci USA 1996; 93 (10): 4897-4902. PMID: 8643500.

7. Fraley R, Subramani S, Berg P, Papahadjopoulos D. Introduction of liposome-encapsulated SV40 DNA into cells. J Biol Chem 1980; 255 : 10431-10435.

8. Halama A, Kulinski M, Librowski T, Lochynski S. Polymer-based non-viral gene delivery as a concept for the treatment of cancer. Pharmacol Rep 2009; 61 (6): 993-999. https://doi.org/10.1016/S1734-1140 (09)70160-4 .

9. Zuris JA, Thompson DB, Shu Y et al. Cationic lipid-mediated delivery of proteins enables efficient protein-based genome editing in vitro and in vivo. Nat Biotechnol 2015; 33 (1): 73-80. DOI: 10.1038/nbt.3081.

10. Felgner JH, Kumar R, Sridhar CN et al. Enhanced gene delivery and mechanism studies with a novel series of cationic lipid formulations. J Biol Chem 1994; 269 (4): 2550-2561. PMID: 8300583.

11. Felgner PL, Tsai YJ, Sukhu L,et al. Improved cationic lipid formulations for in vivo gene therapy. Ann NY Acad Sci 1995; 772: 126-139. PMID: 8546385. 
12. Wallenstein EJ, Barminko J, Schloss RS, Yarmush ML. Serum starvation improves transient transfection efficiency in differentiating embryonic stem cells. Biotechnol Prog 2010; 26 (6): 1714-1723. DOI: 10.1002/btpr.472.

13. Shabani M, Hemmati $S$, Hadavi $R$ et al. Optimization of gene transfection in murine myeloma cell lines using different transfection reagents. Avicenna J Med Biotechnol 2010; 2 (3): 123-130. PMID: 23408356.

14. Rejman J, Bragonzi A, Conese M. Role of clathrin- and caveolaemediated endocytosis in gene transfer mediated by lipo- and polyplexes. Mol Ther 2005; 12 (3): 468-474. DOI: 10.1016/j.ymthe.2005.03.038.

15. Feng G, Hicks P, Chang, PL. Differential expression of mammalian or viral promoter-driven gene in adherent versus suspension cells. In Vitro Cell Dev Biol Anim 2003; 39 (10): 420-423. DOI: 10.1290/1543-706X (2003)039<0420:DEOMOV >2.0.CO;2.

16. Sandbichler AM, Aschberger T, Pelster B. A Method to Evaluate the Efficiency of Transfection Reagents in an Adherent Zebrafish Cell Line. Biores 2013; 2 (1): 20-27. DOI: 10.1089/biores.2012.0287.

17. Peng $\mathbf{L}$, Xiong $\mathbf{W}$, Cai $\mathbf{Y}$ et al. A simple rapid method for evaluation of transfection efficiency based on fluorescent dye. Bioengineered 2017; 8 (3): 225-231. DOI: 10.1080/21655979.2016.1222995.

18. Marjanovič I, Kandušer M, Miklavčič D, Keber MM, Pavlin M. Comparison of flow cytometry, fluorescence microscopy and spectrofluorometry for analysis of gene electrotransfer efficiency. J Membr Biol 2014; 247 (12): 1259-1267. DOI: 10.1007/s00232-014-9714-4.

19. Maurisse R, De Semir D, Emamekhoo H, Bedayat B, Abdolmohammadi A, Parsi $\mathrm{H}$ et al. Comparative transfection of DNA into primary and transformed mammalian cells from different lineages. BMC Biotechnol 2010; 10: 9. DOI: 10.1186/1472-6750-10-9.
20. Martín-Montañez E, López-Téllez JF, Acevedo MJ, Pavía J, Khan ZU. Efficiency of gene transfection reagents in NG108-15, SH-SY5Y and CHO-K1 cell lines. Methods Find Exp Clin Pharmacol 2010; 32 (5): 291-297. DOI: $10.1358 / \mathrm{mf} .2010 .32 .5 .1498327$.

21. Hunt MA., Currie MJ, Robinson BA, Dachs GU. Optimizing transfection of primary human umbilical vein endothelial cells using commercially available chemical transfection reagents. J Biomol Tech 2010; 21 (2): 66-72. PMID: 20592869.

22. Gharaati-Far N, Tohidkia MR, Dehnad A, Omidi, Y. Efficiency and cytotoxicity analysis of cationic lipids-mediated gene transfection into AGS gastric cancer cells. Artif Cells Nanomed Biotechnol 2017; 46 (5): 1001-1008. DOI: 10.1080/21691401.2017.1355311.

23. Yu X, Liang X, Xie H et al. Improved delivery of Cas9 protein/gRNA complexes using lipofectamine CRISPRMAX. Biotechnol Lett 2016; 38 (6): 919-929. DOI: 10.1007/s10529-016-2064-9.

24. Merhi M, Dombu CY, Brient A et al. Study of serum interaction with a cationic nanoparticle: Implications for in vitro endocytosis, cytotoxicity and genotoxicity. Int J Pharm 2012; 423: 37-44. DOI: 10.1016/j. ijpharm.2011.07.014.

25. Smith PJ, Giroud M, Wiggins HL et al. Cellular entry of nanoparticles via serum sensitive clathrin-mediated endocytosis, and plasma membrane permeabilization. Int J Nanomedicine 2012; 7: 2045-2055. DOI: 10.2147/IJN.S29334.

26. Liu H, Ren C, Zhu B et al. High-efficient transfection of human embryonic stem cells by single cell-plating and starvation. Stem cells and Development 2016; 25 (6): 477-491. DOI: 10.1089/scd.2015.0301.

Received July 7, 2018. Accepted August 20, 2018. 